\title{
Influencia de seis sustratos en el crecimiento de Pinus ponderosa producido en contenedores bajo condiciones de invernáculo
}

\author{
Influence of six substrates in Pinus ponderosa grown in containers under greenhouse conditions
}

\author{
Verónica B. Olivo ${ }^{1 *}$, Carlos G. Buduba ${ }^{2}$ \\ *Autor de correspondencia: ${ }^{1}$ Universidad Nacional de la Patagonia San Juan Bosco, Cátedra de Suelos Forestales, \\ C.C. 14, 9200 Esquel, Chubut, Argentina, olivomainetti@hotmail.com \\ 2 cgbuduba@correo.inta.gov.ar
}

\begin{abstract}
SUMMARY
The influence of six substrates on Pinus ponderosa grown was studied in containers under greenhouse conditions. Treatments used were elaborated in a 1:1 proportion with an organic (coco fiber or Sphagnum peat moss) and an inorganic (pumice, vermiculite or perlite) compound. Different morphologic features were measured eight months later. The plants developed on vermiculite showed the best results. The combination of vermiculite with peat moss presented the greater size for all studied variables: stem diameter $(4.9 \mathrm{~cm})$, shoot height $(25.4 \mathrm{~cm})$, wet and dry weight of the whole plant $(20.9$ and $5.7 \mathrm{~g})$, dry weight of the aerial and root portion ( 3.5 and $2.1 \mathrm{~g})$, dry weight of the main root $(0.7 \mathrm{~g})$ and dry weigh of secondary roots ( $1.5 \mathrm{~g})$. Three indexes were also evaluated in order to determine the plant quality. The substrates with vermiculite presented the best behavior for the different indexes.
\end{abstract}

Key words: substrate, growing media, greenhouse, Pinus ponderosa.

\section{RESUMEN}

Se estudió la influencia de seis sustratos en el crecimiento de Pinus ponderosa producido en contenedores bajo condiciones de invernáculo. Los tratamientos utilizados fueron elaborados en una proporción 1:1 con un componente orgánico (fibra de coco o turba de musgo Sphagnum sp.) y otro inorgánico (pumita, vermiculita o perlita). A los ocho meses las plantas desarrolladas sobre vermiculita presentaron los mejores resultados, siendo la combinación con turba la que alcanzó el mayor tamaño en todas las variables estudiadas: diámetro de cuello $(4,9 \mathrm{~cm})$, altura de tallo $(25,4 \mathrm{~cm})$, peso húmedo y seco de toda la planta $(20,9$ y $5,7 \mathrm{~g})$, peso seco de la parte aérea y radical $(3,5$ y $2,1 \mathrm{~g})$, peso seco de la raíz principal $(0,7 \mathrm{~g})$ y peso seco de las raíces secundarias $(1,5 \mathrm{~g})$. Además, se evaluaron tres índices para determinar la calidad de la planta, que mostraron para los sustratos con vermiculita el mejor comportamiento.

Palabras clave: sustrato, medio de crecimiento, invernáculo, Pinus ponderosa.

\section{INTRODUCCIÓN}

En la región Andino Patagónica argentina la producción de plantas destinadas a la forestación con especies de rápido crecimiento se realiza generalmente bajo la modalidad de raíz desnuda, al aire libre y sin control de las variables climáticas y edáficas, obteniéndose un producto a los dos o tres años, dependiendo de la zona y el manejo (Contardi 1999a). Una alternativa utilizada exitosamente en diversos lugares, es la producción bajo condiciones de invernáculo, en donde, al controlar las variables climáticas y edáficas, se puede lograr en ocho meses una planta de igual o mejor calidad que la obtenida con el esquema tradicional (Enricci et al. 2001). Esta disminución en el tiempo de producción representa una importante ventaja, pero exige un adecuado ajuste de todos los factores que afectan el crecimiento. Dentro de estos, los diferentes elementos que componen el sustrato son muy importantes por cumplir un doble rol: soporte y almacén de agua y nutrientes para las plantas.

Los sustratos están compuestos, generalmente, en diferentes proporciones por una mezcla de dos o más materiales (orgánicos, inorgánicos y/o sintéticos), con el objetivo de complementar propiedades adecuadas para el crecimiento vegetal que no poseen en forma independiente (Gallardo 2003). La combinación y proporción de los materiales del sustrato debe ser cuidadosamente estudiada, según los requerimientos de cada especie, pues el volumen limitado de los contenedores exige óptimas propiedades físicas y químicas para el crecimiento (Landis et al. 1990, Gerding et al. 1996, Lavado 2000). 
El presente estudio evalúa la influencia de seis sustratos en el crecimiento de plantas de pino ponderosa (Pinus ponderosa Dougl. ex Laws.) producidas en contenedores, bajo condiciones controladas de invernáculo, a lo largo de un periodo de crecimiento. Esta especie es la más utilizada en la región porque crece vigorosamente, superando incluso los crecimientos observados en su lugar de origen, Oeste de Estados Unidos (Gonda y Cortés 2001).

\section{MÉTODOS}

El ensayo se llevó a cabo en la ciudad de Esquel (42 $54^{\prime}$ S, $71^{\circ} 19^{\prime} \mathrm{O}$, Chubut, Argentina), dentro del invernáculo de la Universidad Nacional de la Patagonia San Juan Bosco, bajo condiciones controladas de temperatura y fertirrigación (Enricci et al. 2001). Se utilizaron contenedores marca Hiko, de la línea "SS V-120", que poseen 40 celdas individuales de $120 \mathrm{~cm}^{3}$ cada una. Para elaborar los sustratos se usaron cinco componentes, los orgánicos fibra de coco y turba de musgo Sphagnum sp., y los inorgánicos pumita, vermiculita y perlita. Estos se mezclaron homogéneamente de a dos, en una proporción volumétrica 1:1, obteniéndose los siguientes seis sustratos: sustrato 1: coco + pumita; sustrato 2: coco + vermiculita; sustrato 3: coco + perlita; sustrato 4: turba + pumita; sustrato 5: turba + vermiculita, y sustrato 6: turba + perlita. Estos fueron dispuestos en 24 bandejas con un diseño completamente aleatorio, en el cual cada uno de los seis sustratos estaba representado por cuatro bandejas o unidades experimentales (repeticiones). Antes de iniciar el cultivo se realizó una caracterización analítica de cada sustrato por duplicado (cuadro 1). En septiembre de 2001 se sembró manualmente con semilla de pino ponderosa, estratificada durante un mes a una temperatura de $4-5^{\circ} \mathrm{C}$, de procedencia INTA Golondrinas, rodal "Las Golondrinas" ( $42^{\circ} 00^{\prime} \mathrm{S}, 71^{\circ} 31^{\prime} \mathrm{O}$ ), tamaño 1 (mayor a 3,75 mm de diámetro), cosecha 2000, primera selección. El ensayo fue sometido a las mismas condiciones de manejo que el resto de la producción y que se encuentran descritas en Enricci et al. (2001).

En mayo de 2002, después de un período de crecimiento de ocho meses, se efectuaron las siguientes mediciones en cada una de las plantas: diámetro de cuello, altura del tallo, peso húmedo de toda la planta, peso seco de toda la planta, peso seco de la parte aérea, peso seco de la parte radical, peso seco de la raíz principal y peso seco de las raíces secundarias (Mexal y Landis 1990, Contardi 1999b). Además, para determinar la calidad de la planta, se utilizaron los siguientes índices:

- Índice tallo raíz (ITR) (Iverson 1984):

$$
\mathrm{ITR}=\frac{\text { peso seco del tallo }(\mathrm{g})}{\text { peso seco de la raíz }(\mathrm{g})}
$$

- Índice de calidad de Dickson (QI) (Dickson et al. 1960):

$$
\mathrm{QI}=\frac{\text { peso seco total }(\mathrm{g})}{\frac{\text { altura tallo }(\mathrm{cm})}{\text { diámetro tallo }(\mathrm{mm})}+\frac{\text { peso seco tallo }(\mathrm{g})}{\text { peso seco raíces }(\mathrm{g})}}
$$

- Índice de esbeltez de Schmidt-Vogt (IE) (SchmidtVogt 1980):

$$
\mathrm{IE}=\frac{\text { diámetro tallo }(\mathrm{mm})}{\frac{\text { altura tallo }(\mathrm{cm})}{10}+2}
$$

En total se analizaron 667 plantas, resultantes de haber descartado aquellas que se presentaban secas al momento de la medición (la mortandad fue homogénea entre sustratos). Además, como las plantas ubicadas al borde de los pasillos de circulación evidenciaron un crecimien-

Cuadro 1. Datos analíticos promedio $(n=2)$ de cada sustrato. $C I C=$ Capacidad de intercambio catiónico; $M O=$ materia orgánica; $\mathrm{DA}=$ densidad aparente; $\mathrm{CE}=$ conductividad eléctrica; $\mathrm{CC}=$ capacidad de campo; $\mathrm{PMP}=$ punto de marchitez permanente; $\mathrm{AU}=$ agua útil. Sustrato: $1=$ coco + pumita; $2=$ coco + vermiculita; $3=\operatorname{coco}+$ perlita; $4=$ turba + pumita; $5=$ turba + vermiculita; $6=$ turba + perlita.

Analytic data of six substrates $(\mathrm{n}=2)$. CIC $=$ cation exchange capacity, $\mathrm{MO}=$ organic matter, $\mathrm{DA}=$ bulk density, $\mathrm{CE}=$ electrical conductivity; $\mathrm{CC}=$ field capacity, $\mathrm{PMP}=$ wilting coefficient and $\mathrm{AU}=$ available water. Substrate: $1=$ coco fiber + pumice, $2=$ coco fiber + vermiculite, $3=$ coco fiber + perlite, $4=$ peat moss + pumice, $5=$ peat moss + vermiculite, $6=$ peat moss + perlite.

\begin{tabular}{cccccccccc}
\hline \multirow{2}{*}{ Sustrato } & $\begin{array}{c}\mathrm{CIC} \\
(\mathrm{meq} / 100 \mathrm{~g})\end{array}$ & $\begin{array}{c}\mathrm{MO} \\
(\%)\end{array}$ & \multicolumn{2}{c}{$\mathrm{pH}(1: 1)$} & $\mathrm{DA}$ & $\mathrm{CE}$ & $\mathrm{CC}$ & $\begin{array}{c}\mathrm{PMP} \\
\left(\% \mathrm{~cm}^{3} / \mathrm{cm}^{3}\right)\end{array}$ & AU \\
\hline 1 & 18,4 & 14,6 & 4,9 & 4,5 & 0,30 & 505 & 11,0 & 6,6 & 4,4 \\
2 & 44,3 & 14,4 & 6,1 & 5,3 & 0,18 & 728 & 13,8 & 8,4 & 5,4 \\
3 & 42,3 & 36,2 & 6,4 & 5,4 & 0,15 & 266 & 13,2 & 8,9 & 4,3 \\
4 & 17,9 & 10,7 & 3,6 & 3,1 & 0,28 & 168 & 9,5 & 5,3 & 4,2 \\
5 & 48,9 & 11,5 & 5,7 & 4,5 & 0,21 & 201 & 21,2 & 14,2 & 7,0 \\
6 & 59,5 & 37,2 & 3,7 & 3,5 & 0,10 & 195 & 13,8 & 8,8 & 5,0 \\
\hline
\end{tabular}


to diferencial ("efecto borde"), no se midieron las que se encontraban en dicha posición. Los datos fueron examinados con un análisis de varianza factorial para dos factores (factor orgánico con dos niveles: coco y turba; factor inorgánico con tres niveles: pumita, vermiculita y perlita). Para evaluar las diferencias entre tratamientos se utilizó la prueba de comparaciones múltiples de Tukey (Mendenhall 1990).

Para los factores orgánico e inorgánico no se detectó interacción $(P>0,05)$ en ninguna de las variables estudiadas (cuadro 2). Como los componentes orgánico e inorgánico no actuaron recíprocamente se estudió cada factor independientemente del otro.

\section{RESULTADOS}

Al considerar el factor orgánico solo, en la turba se observaron los promedios significativamente más altos $(P<0,05)$ en el peso húmedo de toda la planta, el peso seco de la parte radical y el peso seco de las raíces secundarias (cuadro 3). Para la fracción inorgánica se detectaron diferencias significativas $(P<0,05)$ en todas las variables morfológicas analizadas, excepto en el peso seco

Cuadro 2. Valor de F y $P$ para los factores orgánico, inorgánico y la interacción orgánico x inorgánico. Variable: d = diámetro de cuello; $\mathrm{h}=$ altura del tallo; $\mathrm{PH}=$ peso húmedo de toda la planta; $\mathrm{PS}=$ peso seco de toda la planta; PSA = peso seco de la parte aérea; PSR = peso seco de la parte radical; PSRP = peso seco de la raíz principal; PSRS = peso seco de las raíces secundarias; ITR = índice tallo raíz; QI = índice de calidad de Dickson; IE = índice de esbeltez .

$\mathrm{F}$ and $P$ values for organic, inorganic and interaction of organic * inorganic factors. Feature: $\mathrm{d}=$ stem diameter, $\mathrm{h}=$ shoot height, $\mathrm{PH}=$ wet weight of the whole plant, PS = dry weight of the whole plant, PSA = dry weight of the aerial portion, PSR = dry weigh of the root, PSRP = dry weight of the main root, PSRS = dry weigh of secondary roots, ITR = stem root index, QI = Dickson quality index and $\mathrm{IE}=$ slenderness index

\begin{tabular}{|c|c|c|c|c|c|c|}
\hline \multirow[t]{2}{*}{ Variable } & \multicolumn{2}{|c|}{ Orgánico } & \multicolumn{2}{|c|}{ Inorgánico } & \multicolumn{2}{|c|}{$\begin{array}{l}\text { Orgánico x } \\
\text { inorgánico }\end{array}$} \\
\hline & $\mathrm{F}$ & $P$ & $\mathrm{~F}$ & $P$ & $\mathrm{~F}$ & $P$ \\
\hline $\mathrm{d}(\mathrm{mm})$ & 0,11 & 0,75 & 12,08 & 0,00 & 1,06 & 0,37 \\
\hline $\mathrm{h}(\mathrm{cm})$ & 0,23 & 0,64 & 20,07 & 0,00 & 1,87 & 0,18 \\
\hline $\mathrm{PH}(\mathrm{g})$ & 8,56 & 0,01 & 13,45 & 0,00 & 0,69 & 0,52 \\
\hline PS (g) & 0,89 & 0,36 & 30,49 & 0,00 & 1,66 & 0,22 \\
\hline PSA (g) & 0,47 & 0,50 & 49,57 & 0,00 & 3,57 & 0,05 \\
\hline PSR (g) & 5,39 & 0,03 & 7,79 & 0,00 & 0,12 & 0,89 \\
\hline PSRP (g) & 0,09 & 0,77 & 21,22 & 0,00 & 0,58 & 0,57 \\
\hline PSRS (g) & 7,59 & 0,01 & 3,05 & 0,07 & 0,04 & 0,96 \\
\hline ITR & 11,21 & 0,00 & 7,26 & 0,01 & 2,47 & 0,11 \\
\hline QI & 0,26 & 0,62 & 7,96 & 0,00 & 0,47 & 0,63 \\
\hline IE & 1,14 & 0,30 & 2,28 & 0,13 & 0,29 & 0,75 \\
\hline
\end{tabular}

Cuadro 3. Promedio para las variables medidas \pm 1 error estándar (ES) para el factor orgánico (coco, turba) e inorgánico (pumita, vermiculita, perlita). Variable: $\mathrm{d}=$ diámetro de cuello; $\mathrm{h}=$ altura del tallo; $\mathrm{PH}=$ peso húmedo de toda la planta; $\mathrm{PS}=$ peso seco de toda la planta; $P S A=$ peso seco de la parte aérea; $P S R=$ peso seco de la parte radical; PSRP = peso seco de la raíz principal; PSRS = peso seco de las raíces secundarias; ITR = índice tallo raíz; QI = índice de calidad de Dickson; IE = índice de esbeltez. Letras diferentes junto al promedio indican diferencias significativas $(P<0,05)$.

Average for measured variables \pm 1 standard error (ES) for organic (coco fiber, peat moss) and inorganic (pumice, vermiculite, perlite) factor. Feature: $\mathrm{d}=$ stem diameter, $\mathrm{h}=$ shoot height, $\mathrm{PH}=$ wet weight of the whole plant, PS = dry weight of the whole plant, PSA = dry weight of the aerial portion, PSR = dry weigh of the root, PSRP $=$ dry weight of the main root, PSRS $=$ dry weigh of secondary roots, $\mathrm{ITR}=$ stem root index, $\mathrm{QI}=$ Dickson quality index and IE $=$ slenderness index. Different letters indicate significant differences $(P<0.05)$.

\begin{tabular}{|c|c|c|c|c|}
\hline \multirow{3}{*}{ Variable } & \multicolumn{4}{|c|}{ Factor } \\
\hline & \multicolumn{2}{|c|}{ Orgánico $(\mathrm{n}=12)$} & \multicolumn{2}{|c|}{ Inorgánico $(\mathrm{n}=8)$} \\
\hline & Nivel & $\mathrm{X} \pm 1 \mathrm{ES}$ & Nivel & $\mathrm{X} \pm 1 \mathrm{ES}$ \\
\hline \multirow[t]{3}{*}{ d } & Coco & $4,39^{\mathrm{a}} \pm 0,11$ & Pumita & $4,31^{\mathrm{b}} \pm 0,12$ \\
\hline & Turba & $4,35^{\mathrm{a}} \pm 0,16$ & Vermiculita & $4,81^{\mathrm{a}} \pm 0,11$ \\
\hline & & & Perlita & $3,98^{\mathrm{b}} \pm 0,12$ \\
\hline \multirow[t]{3}{*}{$\mathrm{h}$} & Coco & $1,92^{\mathrm{a}} \pm 0,68$ & Pumita & $22,15^{\mathrm{b}} \pm 0,64$ \\
\hline & Turba & $22,22^{\mathrm{a}} \pm 0,82$ & Vermiculita & $24,49^{\mathrm{a}} \pm 0,61$ \\
\hline & & & Perlita & $19,57^{\mathrm{c}} \pm 0,41$ \\
\hline \multirow[t]{3}{*}{$\overline{\mathrm{PH}}$} & Coco & $16,08^{b} \pm 0,71$ & Pumita & $16,90^{\mathrm{b}} \pm 0,88$ \\
\hline & Turba & $18,27^{\mathrm{a}} \pm 0,82$ & Vermiculita & $19,68^{a} \pm 0,82$ \\
\hline & & & Perlita & $14,94^{b} \pm 0,48$ \\
\hline \multirow[t]{3}{*}{ PS } & Coco & $4,52^{\mathrm{a}} \pm 0,18$ & Pumita & $4,60^{\mathrm{b}} \pm 0,15$ \\
\hline & Turba & $4,67^{\mathrm{a}} \pm 0,27$ & Vermiculita & $5,39^{\mathrm{a}} \pm 0,18$ \\
\hline & & & Perlita & $3,79^{\mathrm{c}} \pm 0,11$ \\
\hline \multirow[t]{3}{*}{$\overline{\mathrm{PSA}}$} & Coco & $2,80^{\mathrm{a}} \pm 0,12$ & Pumita & $2,69^{\mathrm{b}} \pm 0,09$ \\
\hline & Turba & $2,73^{\mathrm{a}} \pm 0,19$ & Vermiculita & $3,39^{a} \pm 0,09$ \\
\hline & & & Perlita & $2,22^{\mathrm{c}} \pm 0,10$ \\
\hline \multirow[t]{3}{*}{$\begin{array}{l}\text { PSR } \\
\end{array}$} & Coco & $1,72^{\mathrm{b}} \pm 0,08$ & Pumita & $1,91^{\mathrm{a}} \pm 0,09$ \\
\hline & Turba & $1,94^{\mathrm{a}} \pm 0,09$ & Vermiculita & $2,01^{\mathrm{a}} \pm 0,10$ \\
\hline & & & Perlita & $1,57^{\mathrm{b}} \pm 0,08$ \\
\hline \multirow[t]{3}{*}{ PSRP } & Coco & $0,56^{\mathrm{a}} \pm 0,03$ & Pumita & $0,59^{\mathrm{a}} \pm 0,02$ \\
\hline & Turba & $0,55^{\mathrm{a}} \pm 0,03$ & Vermiculita & $0,64^{\mathrm{a}} \pm 0,03$ \\
\hline & & & Perlita & $0,44^{\mathrm{b}} \pm 0,02$ \\
\hline \multirow[t]{3}{*}{ PSRS } & Coco & $1,15^{\mathrm{b}} \pm 0,06$ & Pumita & $1,32^{\mathrm{a}} \pm 0,08$ \\
\hline & Turba & $1,38^{\mathrm{a}} \pm 0,06$ & Vermiculita & $1,36^{\mathrm{a}} \pm 0,09$ \\
\hline & & & Perlita & $1,12^{\mathrm{a}} \pm 0,07$ \\
\hline \multirow[t]{3}{*}{$\overline{\text { ITR }}$} & Coco & $1,70^{\mathrm{a}} \pm 0,06$ & Pumita & $1,48^{\mathrm{b}} \pm 0,06$ \\
\hline & Turba & $1,47^{\mathrm{b}} \pm 0,07$ & Vermiculita & $1,77^{\mathrm{a}} \pm 0,04$ \\
\hline & & & Perlita & $1,51^{\mathrm{b}} \pm 0,11$ \\
\hline \multirow[t]{3}{*}{$\overline{\mathrm{QI}}$} & Coco & $0,71^{\mathrm{a}} \pm 0,03$ & Pumita & $0,74^{\mathrm{ab}} \pm 0,04$ \\
\hline & Turba & $0,73^{\mathrm{a}} \pm 0,04$ & Vermiculita & $0,82^{\mathrm{a}} \pm 0,04$ \\
\hline & & & Perlita & $0,61^{\mathrm{b}} \pm 0,02$ \\
\hline \multirow[t]{3}{*}{$\mathrm{IE}$} & Coco & $1,06^{\mathrm{a}} \pm 0,02$ & Pumita & $1,04^{\mathrm{a}} \pm 0,03$ \\
\hline & Turba & $1,03^{\mathrm{a}} \pm 0,03$ & Vermiculita & $1,09^{\mathrm{a}} \pm 0,03$ \\
\hline & & & Perlita & $1,00^{\mathrm{a}} \pm 0,02$ \\
\hline
\end{tabular}


Cuadro 4. Promedio para las variables medidas \pm 1 error estándar de cada sustrato. Variable: $\mathrm{d}=$ diámetro de cuello; $\mathrm{h}=$ altura del tallo; $\mathrm{PH}=$ peso húmedo de toda la planta; PS = peso seco de toda la planta; PSA = peso seco de la parte aérea; PSR = peso seco de la parte radical; PSRP = peso seco de la raíz principal; PSRS = peso seco de las raíces secundarias. Sustrato: $1=$ coco + pumita; $2=$ coco + vermiculita; $3=$ coco + perlita $; 4=$ turba + pumita $; 5=$ turba + vermiculita $; 6=$ turba + perlita.

Average for measured variables \pm 1 standard error for each substrate. Feature: $\mathrm{d}=$ stem diameter, $\mathrm{h}=$ shoot height, $\mathrm{PH}=$ wet weight of the whole plant, PS = dry weight of the whole plant, PSA = dry weight of the aerial portion, PSR = dry weigh of the root, PSRP = dry weight of the main root and PSRS $=$ dry weigh of secondary roots. Substrate: $1=$ coco fiber + pumice, $2=$ coco fiber + vermiculite, $3=$ coco fiber + perlite, $4=$ peat moss + pumice, $5=$ peat moss + vermiculite, $6=$ peat moss + perlite.

\begin{tabular}{ccccccccc}
\hline & \multicolumn{7}{c}{ Promedio \pm 1 error estándar $(\mathrm{n}=4)$} \\
\cline { 2 - 9 } Sustrato & $\begin{array}{c}\mathrm{d} \\
(\mathrm{mm})\end{array}$ & $\begin{array}{c}\mathrm{h} \\
(\mathrm{cm})\end{array}$ & $\begin{array}{c}\text { PH } \\
(\mathrm{g})\end{array}$ & $\begin{array}{c}\text { PS } \\
(\mathrm{g})\end{array}$ & $\begin{array}{c}\text { PSA } \\
(\mathrm{g})\end{array}$ & $\begin{array}{c}\text { PSR } \\
(\mathrm{g})\end{array}$ & $\begin{array}{c}\text { PSRP } \\
(\mathrm{g})\end{array}$ & $\begin{array}{c}\text { PSRS } \\
(\mathrm{g})\end{array}$ \\
\hline 1 & $4,4 \pm 0,2$ & $21,3 \pm 1,0$ & $16,2 \pm 1,1$ & $4,6 \pm 0,3$ & $2,7 \pm 0,2$ & $1,8 \pm 0,2$ & $0,6 \pm 0,1$ & $1,2 \pm 0,2$ \\
2 & $4,7 \pm 0,1$ & $23,1 \pm 0,6$ & $18,7 \pm 0,6$ & $5,1 \pm 0,2$ & $3,3 \pm 0,1$ & $1,9 \pm 0,1$ & $0,6 \pm 0,1$ & $1,2 \pm 0,1$ \\
3 & $4,1 \pm 0,2$ & $19,5 \pm 0,9$ & $14,5 \pm 0,7$ & $3,9 \pm 0,2$ & $2,4 \pm 0,2$ & $1,5 \pm 0,1$ & $0,5 \pm 0,0$ & $1,0 \pm 0,1$ \\
4 & $4,3 \pm 0,2$ & $21,6 \pm 0,9$ & $18,4 \pm 0,7$ & $4,6 \pm 0,2$ & $2,6 \pm 0,1$ & $2,0 \pm 0,1$ & $0,6 \pm 0,0$ & $1,4 \pm 0,1$ \\
5 & $4,9 \pm 0,2$ & $25,4 \pm 0,9$ & $20,9 \pm 1,2$ & $5,7 \pm 0,3$ & $3,5 \pm 0,2$ & $2,1 \pm 0,2$ & $0,7 \pm 0,1$ & $1,5 \pm 0,2$ \\
6 & $3,8 \pm 0,2$ & $19,6 \pm 0,3$ & $15,4 \pm 0,7$ & $3,7 \pm 0,2$ & $2,0 \pm 0,1$ & $1,7 \pm 0,1$ & $0,4 \pm 0,1$ & $1,2 \pm 0,1$ \\
\hline
\end{tabular}

Cuadro 5. Promedio de los índices de calidad de plantas \pm 1 error estándar de cada sustrato. Variable: ITR = índice tallo raíz; QI = índice de calidad de Dickson; IE = índice de esbeltez. Sustrato: $1=$ coco + pumita $; 2=$ coco + vermiculita $3=$ coco + perlita; $4=$ turba + pumita $; 5=$ turba + vermiculita $; 6=$ turba + perlita .

Average of plants quality indexes \pm 1 standard error for each substrate. Feature: ITR $=$ stem root index, QI $=$ Dickson quality index and IE $=$ slenderness index. Substrate: $1=$ coco fiber + pumice, $2=$ coco fiber + vermiculite, $3=$ coco fiber + perlite, $4=$ peat moss + pumice, $5=$ peat moss + vermiculite, $6=$ peat moss + perlite.

\begin{tabular}{cccc}
\hline \multirow{2}{*}{ Sustrato } & \multicolumn{3}{c}{ Promedio \pm 1 error estándar $(\mathrm{n}=4)$} \\
\cline { 2 - 4 } & ITR & QI & IE \\
\hline 1 & $1,58 \pm 0,09$ & $0,72 \pm 0,06$ & $1,06 \pm 0,03$ \\
2 & $1,80 \pm 0,03$ & $0,78 \pm 0,04$ & $1,09 \pm 0,04$ \\
3 & $1,72 \pm 0,14$ & $0,62 \pm 0,03$ & $1,04 \pm 0,03$ \\
4 & $1,38 \pm 0,06$ & $0,74 \pm 0,06$ & $1,03 \pm 0,05$ \\
5 & $1,74 \pm 0,09$ & $0,85 \pm 0,07$ & $1,09 \pm 0,04$ \\
6 & $1,29 \pm 0,05$ & $0,59 \pm 0,03$ & $0,97 \pm 0,04$ \\
\hline
\end{tabular}

de las raíces secundarias. Siempre la presencia de vermiculita manifestó los valores más altos en relación a los otros dos componentes inorgánicos (pumita, perlita). En la comparación de los seis sustratos (cuadro 4) se observó que las plantas desarrolladas en el sustrato 5 (turba + vermiculita) obtuvieron mayor tamaño en todas las variables.

Los índices de calidad de plantas presentaron diferencias significativas $(P<0,05)$, para el factor orgánico, en el índice de tallo raíz (ITR). El coco presentó mejor comportamiento en relación a la turba (cuadro 3). Para los componentes inorgánicos la vermiculita mostró diferencias significativas $(P<0,05)$ en el índice tallo raíz (ITR) e índice de calidad de Dickson (QI). Al considerar cada uno de los sustratos (cuadro 5) se destaca que, para el índice tallo raíz (ITR), la mezcla coco + vermiculita (sustrato 2) es la que presentó la media más alta. En el caso del índice de calidad de Dickson (QI), el sustrato 5 (turba + vermiculita) tuvo el valor más elevado.

\section{DISCUSIÓN}

El mejor comportamiento de los sustratos formados con vermiculita puede deberse a que dicho componente inorgánico brindaría las mejores condiciones fisicoquímicas para el crecimiento vegetal. Sin embargo, Landis et al. (1990) señalan que la vermiculita es estructuralmente inestable y, por ello, debe emplearse en mezclas con un componente que provea resistencia a la compactación. En este estudio, la combinación con turba (sustrato 5) resolvió mejor esta debilidad cuando se analizó el peso húmedo de toda la planta, el peso seco de la parte radical y el peso seco de las raíces secundarias.

Según el índice tallo raíz (ITR), los sustratos con coco + pumita, coco + vermiculita, coco + perlita y turba + vermiculita produjeron plantas con valores superiores a 1,5 , presentando los sustratos con vermiculita los más elevados ( 1,80 y 1,74 , respectivamente). Estas cifras indican buena calidad de planta, pues Iverson (1984) señala valores entre 1,5 y 2 como óptimos, variando éstos de acuerdo a la especie analizada. Por otro lado, Dickson et al. (1960) consideran que cuanto más alto es el valor de su índice (QI) mejor es la calidad de la planta; en este estudio ello se logró con la mezcla turba + vermiculita. Para el índice de esbeltez (IE) un valor de uno es adecuado para plantas normales de pino ponderosa (Dengler et al. 1990), similar a lo obtenido con los seis sustratos estudiados. 


\section{CONCLUSIONES}

En general, los sustratos con vermiculita, independientemente del componente orgánico (fibra de coco o turba de musgo Sphagnum sp.), presentaron los mejores resultados, siendo la combinación con turba la que tuvo diferencias más claras con respecto a los demás sustratos.

\section{AGRADECIMIENTOS}

Nuestro agradecimiento por la colaboración brindada a Ludmila La Manna, Jorge Irisarri, Juan Enricci, Eduardo Núñez, Mariela Pasquini, Liliana Contardi, Martín Jiménez, Diego Massone, Ángela Baggio y Leila Espinosa, pertenecientes a la Universidad Nacional de la Patagonia San Juan Bosco.

\section{REFERENCIAS}

Contardi L. 1999a. Producción de plantines de pino ponderosa: estado actual. In Actas V Jornadas Técnicas de Viveristas Forestales de la Patagonia. San Martín de los Andes, Neuquén: 7-9.

Contardi L. 1999b. Medición de plantines. Patagonia Forestal V (3): 11-12.

Dengler A, E Röhrig, HA Gussone. 1990. Waldbau auf ökologischer Grundlage. Zweiter Band. $6^{\mathrm{a}}$ ed. Hamburg y Berlin, Alemania. Paul Parey. 314 p.

Dickson A, AL Leaf, IE Hosner. 1960. Quality appraisal of white spruce and white pine seedlings stock in nurseries. Forest Chronicle 36: 10-13.

Enricci JA, G Alday, D Massone. 2001. Producción de plantines en contenedores. In Actas VI Jornadas Técnicas de
Viveristas Forestales de la Patagonia. UNPSJB, Esquel, Chubut.

Gallardo C. 2003. Materiales comúnmente utilizados en la formulación de sustratos. In Actas Jornada Técnica: "Introducción al uso de sustratos en la producción comercial de plantines de viveros". EEA INTA, Concordia, Entre Ríos: 5-12.

Gerding V, ME Hermosilla, R Grez. 1996. Sustratos de corteza compostada para la propagación vegetativa de estacas de tallo de Podocarpus nubligena Lindl. y Eucryphia cordifolia Cav. Bosque 17(2): 57-64.

Gonda HE, GO Cortés. 2001. Ecuaciones para el manejo de las plantaciones de pino ponderosa en Neuquén. Publicación técnica $\mathrm{N}^{\circ}$ 30. CIEFAP. Esquel, Chubut. 24 p.

Iverson RD. 1984. Planting stock selection: Meeting biological needs and operational realities. In Duryea ML, TD Landis eds. Forest nursery manual. Oregon State University. Corvallis, USA. p. 261-266.

Landis T, RW Tinus, SE McDonald, JP Barnett. 1990. The container tree nursery manual. Handbook 674. Washington, DC. USDA, Forest Service. 85 p.

Lavado R. 2000. Aguas y sustratos para la producción ornamental. Origen, propiedades, manejo, influencia sobre los cultivos y determinaciones. Buenos Aires, Argentina. New Plant. 109 p.

Mendenhall W. 1990. Estadística para administradores. México D. F., México Grupo Ed. Iberoamérica. 817 p.

Mexal JG, TD Landis. 1990. Target seedling concepts: height and diameter. In Target seedling symposium: proceedings, combined meeting of the Western Forest Nursery Associations. Roseburg, Oregon. USDA, Forest Service. 286 p.

Schmidt-Vogt H. 1980. Characterization of plant material, IUFRO Meeting. S1.05-04. In Röhring E, Gussone HA. Waldbau. Zweiter band. Sechste Auflage, Neubearbeitet. Hamburg und Berlin, 1990. 314 p.

Recibido: 24.11 .03

Aceptado: 23.10.06 